\title{
A THEOREM ON MAXIMUM MODULUS ${ }^{1}$
}

\section{ANATOLE BECK}

Introduction. If $D$ is a domain in the plane of complex numbers, then every analytic function achieves its maximum modulus only at the boundary. We phrase this by asserting that if $f$ is analytic in $D$, and $f$ is not constant, and if $x \in \bar{D}$ has the property that

$$
\limsup _{x}|f|=\sup _{D}|f| \text {, }
$$

then $x \in \delta(D)$, the boundary of $D$. If $x$ is the only point for which the above identity holds, then $x$ is the peak point for $f$ in $D$. If $x$ is the peak point for a bounded analytic function in $D$, then $x$ is $a$ peak point of $D$. We are interested in knowing which boundary points of $D$ can be peak points.

W. Rudin [1] defines a boundary point $x$ of a domain $D$ as a removable boundary point if every function bounded and analytic in $D$ can be continued at $x$. All boundary points which are not removable are essential.

We shall show that a point is a peak point of $D$ if and only if it is an essential boundary point.

Lemma 1. If $D$ is a simply connected domain and $x \in \delta(D)$ is linearly accessible from the interior, then there is a schlicht mapping $f$ of $D$ into the open unit disc such that $\lim _{z \rightarrow x} f(z)=1$ and $\lim \sup _{\xi}|f|<1$ if $\xi \in \delta(D), \xi \neq x$.

Proof. Let $(x, x+\alpha]$ be a line segment contained in $D$. Then the function

$$
f(z)=g^{-1}\left(2+4\left(\alpha^{-1}(z-x)\right)^{1 / 2}\right),
$$

where $g(z)=z+1 / z,|z|<1$, is a schlicht function with the properties:

1. $\lim _{z \rightarrow x} f(z)=1$,

2. $|x-y|>\epsilon, y \in D \Rightarrow 1-|f(y)|>\eta=\eta(\epsilon, D)>0$, as we shall show. Let $x_{i} \rightarrow x, x_{i} \in D$ and $|y-x|>\epsilon$. If we examine the mapping $z \rightarrow f_{1}(z)=\alpha^{-1}(z-x)$, it is a linear mapping taking $[x, x+\alpha]$ onto $[0,1] . \alpha^{-1}\left(x_{i}-x\right) \rightarrow 0,\left|f_{1}(y)-0\right|>\epsilon /|\alpha|$. Since $D$, and thus $f_{1}(D)$, is simply connected, we can apply a square root, with $(+1)^{1 / 2}$ $=+1$, and get $f_{2}(z)=\left(f_{1}(z)\right)^{1 / 2},\left(\alpha^{-1}\left(x_{i}-x\right)\right)^{1 / 2} \rightarrow 0$, and

Received by the editors February 13, 1963.

1 This research was supported by the University of Wisconsin under contract No. AF 49(638)-868 with the Air Force Office of Scientific Research. 


$$
\left|f_{2}(y)-0\right|=\left|\left(f_{1}(y)\right)^{1 / 2}\right|=\left(\left|f_{1}(y)-0\right|\right)^{1 / 2}>\left(\frac{\epsilon}{|\alpha|}\right)^{1 / 2}=\epsilon_{1} .
$$

We note that each point of $(0,1]$ is an interior point of $f_{2}(D)$, so that each point of $[-1,0)$ is an exterior point of $f_{2}(D)$. Thus, $\mathrm{Cl}\left[f_{2}(D)\right]$ meets $[-1,0]$ only at 0 . Set $\eta_{1}=\eta_{1}(\epsilon, D)=d\left([-1,0], f_{2}(D)-N\left(0, \epsilon_{1}\right)\right)$ $>0$. Then $d\left(f_{2}(y),[-1,0]\right) \geqq \eta_{1}$. Setting $f_{3}(z)=2+4\left(f_{2}(z)\right)$, we have $f_{3}\left(x_{i}\right) \rightarrow 2$ and $d\left(f_{3}(y),[-2,2]\right) \geqq 4 \eta_{1}$. We now observe that for our function $g, g(z) \rightarrow 2$ iff $z \rightarrow 1$ and $|z| \leqq 1-\epsilon \Rightarrow d(g(z),[-2,2])>\epsilon^{2} .^{2}$ Thus $f\left(x_{i}\right)=g^{-1}\left(f_{3}\left(x_{i}\right)\right)=g^{-1}\left(2+4\left(\alpha^{-1}\left(x_{i}-x\right)\right)^{1 / 2}\right) \rightarrow 1$ and $|f(y)|$ $=\left|g^{-1}\left(f_{3}(y)\right)\right|<1-\left(4 \eta_{1}\right)^{1 / 2}$ so that $1-|f(y)|>\left(4 \eta_{1}\right)^{1 / 2}=\eta(\epsilon, D)>0$, as promised.

Lemma 2. The set of peak points of any domain is closed.

Proof. Let $a_{i}$ be the peak point of $f_{i}$ in $D, i=1,2, \cdots$, and let $a_{i} \rightarrow a$. We assume that $\sup _{D}\left|f_{i}\right|=1$. Let $N_{i}$ be a neighborhood of $a_{i}$, $i=1,2, \cdots$, with $\operatorname{diam}\left(N_{i}\right) \rightarrow 0$ and pairwise disjoint. Since $a_{i}$ is a peak point of $f_{i}, f_{i}$ is bounded away from 1 off $N_{i}$, so that for an appropriate integer $m_{i}$, we have

$$
\sup _{z \in D-N_{i}}\left|f_{i}^{m_{i}}(z)\right|<4^{-i}
$$

Set $g_{i}(z)=f_{i}^{m_{i}}(z)$. We will now generate (inductively) a sequence $\left\{b_{i}\right\}$ such that

1. $\left|b_{i}\right| \leqq 2$,

2. $\sum_{i=1}^{\infty} b_{i} g_{i}$ is bounded and analytic in $D$,

3. $\sup _{N_{n}}\left|\sum_{i=1}^{n} b_{i} g_{i}\right|=2-1 / n$.

We take $b_{1}=1$. Given $b_{1}, \cdots, b_{n-1}$, we find $b_{n}$ as follows:

Set $h_{n}(\xi)=\sup _{N_{n}}\left|b_{1} g_{1}+\cdots+b_{n-1} g_{n-1}+\xi \cdot g_{n}\right|,|\xi| \leqq 2$. Then $h_{n}(0)$ $=\sup _{N_{n}}\left|b_{1} g_{1}+\cdots+b_{n-1} g_{n-1}\right|<\sum_{i=1}^{n-1} 2 \cdot 4^{-i}<2 / 3$. We now choose a point $y_{n}$ such that $g_{n}\left(y_{n}\right)>1-(1 / 2 n)$, and set

$$
c_{n}=2 \frac{b_{1} g_{1}\left(y_{n}\right)+\cdots+b_{n-1} g_{n-1}\left(y_{n}\right)}{g_{n}\left(y_{n}\right)} \cdot\left|\frac{g_{n}\left(y_{n}\right)}{b_{1} g_{1}\left(y_{n}\right)+\cdots+b_{n-1} g_{n-1}\left(y_{n}\right)}\right| \text {. }
$$

Then

$$
\begin{aligned}
h_{n}\left(c_{n}\right) & \geqq\left|b_{1} g_{1}\left(y_{n}\right)+\cdots+b_{n-1} g_{n-1}\left(y_{n}\right)+c_{n} g_{n}\left(y_{n}\right)\right| \\
& \geqq\left|c_{n} g_{n}\left(y_{n}\right)\right|>2-\frac{1}{n},
\end{aligned}
$$

2 This is because the image under $g^{-1}$ of the circle $x^{2}+y^{2}=c^{2}$ is the ellipse $\left(u^{2} / a^{2}\right)$ $+\left(v^{2} / b^{2}\right)=1$, where $a=c+1 / c, b=c-1 / c$. If $c=1-\epsilon$ then the image of $x^{2}+y^{2}<(1-\epsilon)^{2}$ is the exterior of the indicated ellipse. The closest approach of this set to the line $[-2,2]$ is at the vertex, where the distance is $\epsilon^{2} /(1-\epsilon)$. 
since $\sum_{i=1}^{n-1} b_{i} g_{i}\left(y_{n}\right)$ and $c_{n} g_{n}\left(y_{n}\right)$ have the same argument. Since $h_{n}(0)<2 / 3<2-1 / n<h_{n}\left(c_{n}\right)$, there is a point $b_{n}$ in the disc $|z| \leqq 2$ such that $h_{n}\left(b_{n}\right)=2-1 / n$. For $z \in N_{n}$, we then have

$$
\begin{aligned}
\left|\sum_{i=1}^{\infty} b_{i} g_{i}(z)\right| & \leqq\left|\sum_{i=1}^{n} b_{i} g_{i}(z)\right|+\sum_{i=n+1}^{\infty}\left|b_{i}\right| \cdot\left|g_{i}(z)\right| \\
& <2-\frac{1}{n}+\sum_{i=n+1}^{\infty} 2 \cdot 4^{-i}=2-\frac{1}{n}+\frac{2}{3} \cdot 4^{-n} .
\end{aligned}
$$

Similarly,

$$
\sup _{z \in N_{n}}\left|\sum_{i=1}^{\infty} b_{i} g_{i}(z)\right|>2-\frac{1}{n}-\frac{2}{3} \cdot 4^{-n} .
$$

For $z \in D-\bigcup_{i=1}^{\infty} N_{i}$, we have

$$
\left|\sum_{i=1}^{\infty} b_{i} g_{i}(z)\right|<\sum_{i=1}^{\infty} 2 \cdot 4^{-i}=2 / 3 .
$$

Thus $\left|\sum_{i=1}^{\infty} b_{i} g_{i}(z)\right|<2$ for all $z \in D$. If $K$ is a compact subset of $D$, then $K$ meets only finitely many $N_{i}$, so that the series converges uniformly and absolutely on $K$. Thus $f(z)=\sum_{i=1}^{\infty} b_{i} g_{i}(z)$ is analytic. Finally, it is clear that $|f(z)|$ is close to 2 only inside $N_{i}$, for large $i$, that is, only around $a$.

Lemмa 3. If $D$ is any domain, the boundary points linearly accessible from the interior are dense in $\delta(D)$.

Proof. Let $x \in \delta(D), \epsilon>0$. Let $y \in D,|y-x|<\epsilon$. Let $x_{1}$ be the point of $\delta(D) \cap[x, y]$ lying nearest to $y$. Then $\left|x-x_{1}\right|<\epsilon$ and $x_{1}$ is linearly accessible.

THEOREM 1. If $D$ is simply connected, and its boundary consists of more than one point, then every boundary point is a peak point.

Proof. Direct consequence of Lemmas 1, 2, 3.

Lemma 4. If $x \in \delta(D)$ and the component of $x$ in $D^{\prime}, K(x) \neq\{x\}$, then $x$ is a peak point of $D$.

Proof. Let $y \in K(x), y \neq x$. Let $K_{1}$ be a sub-continuum of $K(x)$, containing $y$ but not containing $x$. There is a conformal mapping $\phi$ of $K_{1}^{\prime}$ onto the open unit disc, and $\phi$ is a homeomorphism around $x$. Then $\phi\left(K(x)^{\prime}\right)$ is a simply connected domain, and each linearly accessible point of $\delta\left(\phi\left(K(x)^{\prime}\right)\right)$ is a peak point of the kind described in Lemma 1. If we now restrict our functions to $\phi(D)$, each of these points is still a peak point, though the point may no longer be linearly accessible. Now, by Lemma $2, x$ is a peak point. 
LEMma 5. If $K(D)$ denotes the closure of the union of those components of $\delta(D)$ which are not single points, then every point in $K(D)$ is a peak point of $D$.

Proof. Clear from Lemmas 4 and 2.

Definition. A set $S$ is a Painlevé null set (called a p-null set) if the algebra of bounded analytic functions on $S^{\prime}$ consists of the constants alone.

Definition. A point $x \in \delta(D)$ is called a p-essential boundary point if for each $\epsilon>0, N(x, \epsilon) \cap \delta(D)$ is not a $p$-null set.

Lemma 6. Let $x \in \delta(D)$ and $x \in K(D)$. Then if $x$ is a p-essential boundary point, $x$ is a peak point of $D$.

Proof. Let $N$ be a neighborhood of $x$ in which $\delta(D)$ is totally disconnected. Since the $p$-essential boundary points form a perfect set, let $x_{i} \rightarrow x$ be a sequence of $p$-essential boundary points. Let $N_{i}$ be a sequence of neighborhoods with $N_{i} \subset N, x_{i} \in N_{i}$ no two $N_{i}$ intersecting, and $\operatorname{diam}\left(N_{i}\right) \rightarrow 0$. Let $M_{i}$ be open with $x_{i} \in M_{i} \subset \bar{M}_{i} \subset N_{i}$ for each $i$. Then $K_{i}=\bar{M}_{i} \cap \delta(D)$ is not a $p$-null set and thus we can find $f_{i}$ such that $f_{i}$ is analytic on $K_{i}^{\prime}$ (including $\infty$ ) and $f_{i}$ is not constant. We can assume that $\sup _{K_{i}^{\prime}}|f|=1$. Then $\sup _{N_{i}^{\prime}}\left|f_{i}\right|<1$, so we can choose a sequence of integers $m_{i}$ so that

$$
\sup _{N_{i}^{\prime}}\left|f_{i}^{m_{i}}\right|<4^{-i}
$$

Set $g_{i}(z)=f_{i}^{m_{i}}(z)$. Since $D^{\prime}$ is nowhere dense in $N_{i}$, $\sup _{D}\left|g_{i}\right|=1$, and $\sup _{D-N_{i}}\left|g_{i}\right|<4^{-i}$.

Using the same technique as in Lemma 2 , we choose $b_{n}$ so that

1. $\left|b_{n}\right| \leqq 2$,

2. $\sup _{N_{i}}\left|\sum_{i=1}^{n} b_{i} g_{i}\right|=2-1 / n$,

3. $f(z)=\sum_{i=1}^{\infty} b_{i} g_{i}(z)$ is bounded and analytic in $D$.

Furthermore, we deduce that

$$
2-\frac{1}{n}-\frac{2}{3} \cdot 4^{-n}<\sup _{N_{n}}|f|<2-\frac{1}{n}+\frac{2}{3} \cdot 4^{-n},
$$

and $\sup _{D-U_{i} N_{i}}|f|<2 / 3$. Thus, $f$ has a peak at $x$.

Theorem 2. If $x$ is a p-essential boundary point, then $x$ is a peak point.

Proof. If $x \in K(D)$, this is true by Lemma 5 ; otherwise by Lemma 6.

THEOREM 3. $x$ is a peak point iff $x$ is an essential boundary point. 
Proof. By a theorem of W. Rudin [1], $x$ is an essential boundary point iff $x$ is a $p$-essential boundary point. From the remarks above, every $x \in K(D)$ is a peak point of a function which has no limit at $x$. Thus, no point of $K(D)$ is removable. If $x \in \delta(D)-K(D)$ and $x$ is removable, then each $f$ is continuable there. Since $\delta(D)$ is nowhere dense around $x$, this extension does not change the maximum modulus nor the lim sup at $x$. By the maximum modulus theorem, $f(x)$ is not the maximum. By continuity, $x$ is not a peak for any $f$.

It would be interesting to know when $x$ is the peak point of a bounded analytic function $f$ which has a limit at $x$, or whose modulus has a limit at $x$. It is clear from previous remarks that every essential boundary point is the peak point of a function which does not have these limits. This question is open.

\section{REFERENCE}

1. W. Rudin, Some theorems on bounded analytic functions, Trans. Amer. Math. Soc. 78 (1955), 333-342.

UNIVERSITY OF WISCONSIN 